\title{
Enhanced Hemostatic Performance of Tranexamic Acid-Loaded Chitosan/Alginate Composite Microparticles
}

\author{
Donghong Li, Pengxi Li, Jiatao Zang, and Jiancang Liu \\ State Key Laboratory of Trauma, Burns, and Combined Injury, Second Department of Research Institute of Surgery, \\ Daping Hospital, Third Military Medical University, Chongqing 400042, China
}

Correspondence should be addressed to Donghong Li, lqs_cq@yahoo.com.cn

Received 21 March 2012; Accepted 1 October 2012

Academic Editor: Bernd H. A. Rehm

Copyright (๑) 2012 Donghong Li et al. This is an open access article distributed under the Creative Commons Attribution License, which permits unrestricted use, distribution, and reproduction in any medium, provided the original work is properly cited.

Novel microparticles based on chitosan and sodium alginate were prepared using emulsification and cross-linking technologies. The spherical microparticles had a porous surface and a diameter of $2 \sim 40 \mu \mathrm{m}$. In simulated body fluid, these microparticles quickly swelled but gradually degraded. The results of the MTT assay revealed that a slight inhibition of cell proliferation was observed on day 2 and then gradually decreased afterward. No cell morphology changes were observed. By loading tranexamic acid, the hemostatic performance of the microparticles was obviously improved. Using fast-acting styptic powder (Flashclot) as the control, the hemostatic efficiency was investigated in rabbits using a liver transection bleeding model. It was found that both Flashclot and the microparticles achieved hemostasis in $3.07 \pm 0.84 \mathrm{~min}$ and $2.48 \pm 0.88 \mathrm{~min}$, respectively; however, the tranexamic acid-loaded microparticles stopped the bleeding in $1.90 \pm 0.75 \mathrm{~min}(P<0.05)$. Additionally, Flashclot resulted in heat injury to the experimental livers, while the microparticles did not. Thus, with their biodegradability, safety, and superior hemostatic efficiency, tranexamic acid-loaded microparticles might be a promising new powdered hemostatic agent with a wide range of potential applications.

\section{Introduction}

Massive bleeding is the leading cause of shock and death in combat, transportation accidents, natural disasters, emergencies, and surgeries $[1,2]$. The early identification and surgical control of hemorrhaging is the crucial first step in the management of an injured patient; however, this objective often remains challenging in the most critical trauma patients $[3,4]$. In recent years, an abundance of local hemostatic agents have become commercially available, but most of them are effective only for mild to moderate bleeding. The rapid deployment hemostat $(\mathrm{RDH})$ and the fibrin sealant dressing (FSD) are recognized for their effect in stopping massive vascular bleeding $[5,6]$. However, the $\mathrm{RDH}$ has a complicated production process and unstable efficiency, and the FSD is expensive and has a risk of disease transmission because it is derived from plasma products obtained from pooled donors [7].

Quickclot is a typical composite zeolite hemostatic material that was developed by Z-Medica in 2002 [8]. It was launched into the market with the approval of the US Food and Drug Administration, and has been used in Afghanistan and Iraq. It is superior to traditional hemostatic materials in terms of hemostatic efficiency and survival rate $[9,10]$. As a powdery hemostatic agent, it can be applied to wounds of any shape and depth, which often cannot be reached by traditional hemostatic methods or bandages. Its convenience and ease of portability and storage also make Quickclot a very attractive alternative. On the other hand, one major issue with Quickclot is that heat is generated when absorbing water (reaching about $100^{\circ} \mathrm{C}$ in a short time), which can lead to burns and injuries in peripheral skin, muscles, nerves, vessels, and tissues [11]. It has been reported that in the Iraq war, some wounded US patients had grade II burns because of application of Quickclot [12]. Therefore, in order to reduce heat generation, the dry powder must be removed and the wound washed with a larger than normal volume of water during debridement, which is often inconvenient for following treatments. 
This situation spurred the question of whether a powdery hemostatic agent could be prepared from biomaterials which could generate less heat when absorbing water. It is well known that polysaccharides, such as chitosan and alginate, are cost-effective, biodegradable, and biocompatible. Interestingly, chitosan has several biologic activities such as bacteriostasis [13-15], promotion of wound healing [16, 17], and haemostasis [18-20], and sodium alginate has a very high water absorption rate [21] Additionally, they can be easily manipulated into microparticles [22]. In this study, the preparation of spherical microparticles with a porous surface based on sodium alginate and chitosan was examined. The morphology, water uptake ability, degradation behavior, and cytotoxicity of the microparticles were investigated. Further, in order to improve the hemostatic efficiency of the microparticles, a local hemostatic agent, tranexamic acid, was loaded into it. The drug-release ability and hemostatic performance of the tranexamic acid-loaded microparticles were evaluated to confirm the applicability of this microparticle as a powdery hemostatic agent.

\section{Experimental}

2.1. Materials. The following materials were obtained from the indicated suppliers and used as received. Chitosan (MW $2.5 \times 10^{5}, 85 \%$ degree of deacetylation) was purchased from Kangduo Ocean Biomedical Co. Ltd (Taizhou, China). Sodium alginate (MW $4.2 \times 10^{6}$ ) was purchased from Qingdao Crystal Rock Biology Development Co. Ltd (China). RPMI-1640 culture medium and fetal calf serum were purchased from Hyclone (Utah, USA); trypsin, dimethylsulfoxide (DMSO), and 3-(4,5-dimethylthiazol-2-yl)-2,5-diphenyl tetrazolium bromide (MTT) were purchased from Sigma (USA). Cell culture cluster dishes (96 and 6 well plates) were purchased from Costar (USA). Flashclot was obtained from Honghua Pharmaceutical Co. Ltd (Shenzhen, China). All of the reagents and solvents used were of analytical grade and used without further purification. L929 mouse fibroblast cells were obtained from Shanghai Institute of Cell Biology, Chinese Academy of Sciences.

The preparation of simulated body fluid (SBF) was as follows [23]: $8.035 \mathrm{~g}$ of $\mathrm{NaCl}, 0.355 \mathrm{~g}$ of $\mathrm{NaHCO}_{3}, 0.225 \mathrm{~g}$ of $\mathrm{KCl}, 0.231 \mathrm{~g}$ of $\mathrm{K}_{2} \mathrm{HPO}_{4} \cdot 3 \mathrm{H}_{2} \mathrm{O}, 0.311 \mathrm{~g}$ of $\mathrm{MgCl}_{2} \cdot 6 \mathrm{H}_{2} \mathrm{O}$, $39 \mathrm{~mL}$ of $1.0 \mathrm{M} \mathrm{HCl}, 0.292 \mathrm{~g}$ of $\mathrm{CaCl}_{2}, 0.072 \mathrm{~g}$ of $\mathrm{Na}_{2} \mathrm{SO}_{4}$, and $6.118 \mathrm{~g}$ of Tris were added to $900 \mathrm{~mL}$ of deionized water in a plastic beaker. After the $\mathrm{pH}$ value was adjusted to 7.45 with $1.0 \mathrm{M} \mathrm{HCl}$, the solution was poured into a $1000 \mathrm{~mL}$ volumetric flask, and deionized water was added to a final volume of $1000 \mathrm{~mL}$.

2.2. Fabrication of the Microparticles. Alginate/chitosan microparticles were prepared using the methods of emulsification and crosslinking [24]. Briefly, a $3 \%(w / v)$ chitosan solution was prepared by dissolving chitosan in a $1.5 \%$ acetic acid aqueous solution. A $6 \%(\mathrm{w} / \mathrm{v})$ alginate solution was prepared by dissolving alginate in deionized water. Following the mixing of both solutions, Tween 80 was added, and then the mixture was vigorously stirred to form a uniform white batter. The mixture was then added drop-wise to petroleum ether containing Span 80 and calcium chloride. The final ratio of alginate: chitosan: Tween 80 : Span $80:$ calcium chloride was $3: 3: 2: 2: 1(\mathrm{w} / \mathrm{w} / \mathrm{w} / \mathrm{w} / \mathrm{w})$, and the ratio of water: petroleum ether was $1: 10$. After stirring at $900 \mathrm{rpm}$ for $2 \mathrm{~h}$, the mixture was centrifuged at $4000 \mathrm{~g}$ for $20 \mathrm{~min}$ and washed with $50 \%(\mathrm{v} / \mathrm{v})$ ethanol until no oil spill was observed in the supernatant liquid. After lyophilization, the microparticles were obtained in a yield of $80 \%$.

2.3. Characterization. The morphology of the microparticles was examined with scanning electron microscopy (SEM). The sample was coated with gold (ca. $50 \mathrm{~nm}$ in thickness) using a Hitachi coating unit (IB-2 coater) under high vacuum and then observed with TESCAN VEGA II SEM (Oxford) at an accelerating voltage of $20 \mathrm{kV}$. The size distribution of the microparticles was analyzed using a Rise2000 Powder Diameter Analyzer (Runzhi Technology Co. Ltd, China) after the suspension of the microparticles in deionized water was sonicated for $60 \mathrm{~s}$. The infrared (IR) spectra were recorded on a Varian 640 FT-IR spectrometer (American Perkin Elmer Co.). To exclude the influence of water, the samples and $\mathrm{KBr}$ were fully dried prior to submission of samples to FTIR analyses. The spectra were collected over the range of $4000-500 \mathrm{~cm}^{-1}$.

2.4. Water Sorption Studies. The water sorption capacity of the microparticles was investigated by soaking with SBF. After drying and carefully weighing the samples, they were immersed in 6-well culture plates containing $10 \mathrm{~mL}$ of SBF $(\mathrm{pH}$ 7.4) at room temperature. Subsequently, the samples were withdrawn from the solution after soaking for 2, 4, $6,10,20,30$, or $60 \mathrm{~min}$ and were placed on a glass plate which formed an angle of $45^{\circ}$ from level for $1 \mathrm{~min}$ to remove the redundant solution. The swelled samples were then weighed immediately. The water uptake ratio (WR) of the microparticles in SBF was calculated as follows: WR = $\left(\left(W_{1}-W_{0}\right) / W_{0}\right) \times 100 \%$, where $W_{1}$ denotes the weight of the test sample at a given time during swelling and $W_{0}$ is its initial weight. Three parallel replications were carried out, and the values were expressed as mean \pm standard deviation.

2.5. In Vitro Degradation. The in vitro degradations of the microparticles were also conducted in SBF. Before the experiments, the microparticles were sterilized using Co60 irradiation, and the SBF was filtered with a $0.22 \mu \mathrm{m}$ sterile membrane. Then the preweighed test samples were incubated in $10 \mathrm{~mL}$ of $\mathrm{SBF}$ at $37^{\circ} \mathrm{C}$. At predetermined time intervals, the samples were taken out, washed with deionized water to remove any salt remaining on the surface, and freeze-dried to a constant weight for weight measurement. The weight loss ratios of the samples were calculated using the following equation: degradation rate $(\%)=\left(W_{\text {before }}-\right.$ $\left.W_{\text {after }}\right) / W_{\text {before }} \times 100 \%$, where $W_{\text {before }}$ and $W_{\text {after }}$ were the dry weights of the samples before and after degradation. The values were expressed as mean \pm standard deviation $(n=3)$. 
2.6. Cytotoxicity Study. According to national criteria, the cytotoxicity of the composite microparticles was initially evaluated using the MTT assay [25]. Simply, $150 \mathrm{mg}$ of microparticles were immersed in $7 \mathrm{~mL}$ of RPMI-1640 medium supplemented with $10 \%(\mathrm{v} / \mathrm{v})$ heat-inactivated fetal calf serum and $1 \%(\mathrm{v} / \mathrm{v})$ penicillin-streptomycin $(100$ $\mathrm{U} / \mathrm{mL}$ penicillin $\mathrm{G}$ and $100 \mu \mathrm{g} / \mathrm{mL}$ streptomycin) at room temperature for $24 \mathrm{~h}$. After filtering with a $0.22 \mu \mathrm{m}$ sterile membrane, the extract was immediately used for the MTT assay. There were three experimental groups: (1) the pure extract (100\% concentration), (2) the extract diluted with an equal volume of culture medium (50\% concentration), and (3) the pure culture medium (control group). The L929 cells were cultured for two days and then dissociated from the plate surface using $0.25 \%$ trypsin and transferred to the wells of 96 -well culture plates at $5 \times 10^{3}$ cells/well. After the cells were cultured for $24 \mathrm{~h}$ at $37^{\circ} \mathrm{C}$, the culture medium was removed, and $100 \mu \mathrm{L}$ of the medium with different concentrations of extract was added to the wells. Each group was added into eight wells. After 2, 4, and 7 days, the medium was aspirated and $20 \mu \mathrm{L}$ of MTT stock solution ( $5 \mathrm{mg}$ in $1 \mathrm{~mL}$ of PBS, pH 7.4) was added to each well. After $4 \mathrm{~h}$ of incubation, the medium was removed by aspiration, and $150 \mu \mathrm{L}$ of DMSO was added to each well and the culture plates were rocked for $10 \mathrm{~min}$. The absorbance of the sample at $492 \mathrm{~nm}$ was determined in a Multiskan Spectrum (Thermo) compared with a DMSO blank. The relative cell viability (\%) related to the control was calculated by $[A]$ test $/[A]$ control $\times 100 \%$.

Cell morphology was also observed. The L929 cells were transferred to the wells of 6-well culture plates. After the cells were cultured with the extract (100\% concentration) of the microparticles for 2, 4, and 7 days, the morphology of the L929 cells was observed with an inverted microscope (Olympus, CK40).

2.7. Tranexamic Acid Incorporation. Tranexamic acid powder was dissolved in distilled water, which soaked all the microparticles and was completely absorbed by them. After lyophilization, the final powdery hemostatic agent was obtained containing $2 \%(\mathrm{w} / \mathrm{w})$ tranexamic acid. Then, the microparticles and the tranexamic acid-loaded microparticles were sterilized by Co60 irradiation and were used in the hemostasis assay.

2.8. Tranexamic Acid Release Study. The tranexamic acidloaded microparticles were immersed in PBS ( $\mathrm{pH}$ 7.4) at $37^{\circ} \mathrm{C}$ under gentle shaking. Next, $50 \mu \mathrm{L}$ of solution was withdrawn from the test medium at fixed time intervals and the concentration of tranexamic acid was determined using a 1200 HPLC analyzer (Agilent, USA. $250 \times 4.6 \mathrm{~mm}$ Zorbax 300 Extend-C18 column, $25^{\circ} \mathrm{C}$, mobile phase: $5 \%$ acetonitrile containing $0.1 \%$ tetrahydrofuran (THF), flowing rate: $1 \mathrm{~mL} / \mathrm{min}$, detected wavelength: $254 \mathrm{~nm}$, sample size $1 \mu \mathrm{L}$ ). An equivalent volume of fresh PBS was added to the cells after each sampling to maintain a constant medium volume. Three parallel replicates of the tranexamic acid-loaded microparticles were carried out, and the values were expressed as mean \pm standard deviation.

The tranexamic acid release experiment was also carried out in anticoagulated blood plasma. First, the anticoagulated blood was collected from the femoral artery of rabbits $5 \mathrm{~min}$ after the injection of heparin $(300 \mathrm{u} / \mathrm{kg})$. The red cells were then removed by centrifugation. Then, the tranexamic acid-loaded microparticles were incubated in anticoagulated blood plasma at $37^{\circ} \mathrm{C}$ with gentle shaking; $200 \mu \mathrm{L}$ of solution was withdrawn from the test medium at fixed time intervals, and $100 \mu \mathrm{L}$ of $10 \% \mathrm{HClO}_{4}$ was added to the solution. Following centrifugation at $12000 \mathrm{rpm}$ for $10 \mathrm{~min}$, the concentration of tranexamic acid in supernatant was determined by HPLC as described above.

2.9. Hemostasis Assay. All animals were obtained from the Experimental Animal Center of Third Military Medical University (China). This study was approved by the Research Council and Animal Care and Use Committee of the Research Institute of Surgery, Daping Hospital, Third Military Medical University (Chongqing, China). Animals received care in strict compliance with The Guide for the Care and Use of Laboratory Animals published by the US National institutes of Health (NIH Publication No. 85-23, revised 1996). All efforts were made to minimize suffering and the number of animals used.

Eighteen New Zealand white rabbits, weighing 2.5 $3.0 \mathrm{~kg}$, both female and male, were fasted $12 \mathrm{~h}$ prior to the experiment with free access to water. On the day of the experiment, the rabbits were anaesthetized using intravenous sodium pentobarbital $(50 \mathrm{mg} / \mathrm{kg})$ and then strapped onto a surgical board. After the surgical area was shaved with an electric razor, surgical catheters were inserted into the right femoral arteries of the rabbits. The mean arterial pressure (MAP) was monitored from the cannulated femoral arteries during the surgery. Blood samples were collected from the femoral arteries before and after surgery. The red blood cell (RBC) count, hemoglobin concentration, white blood cell (WBC) count, and platelet count were measured with an XT-1800 auto hematology analyzer (Japan). The activated partial thromboplastin time (APTT) and prothrombin time (PT) were measured with a Beckman Coulter ACL TOP automated coagulation analyzer (USA). Then, a ventral midline incision (approximately $20 \mathrm{~cm}$ long) was made, and the liver was inspected. To create active bleeding injury, the anterior extremity of the right, left, and medial lobes of the liver were cut off with scissors, such that the lobar branches of the portal vein, hepatic vein, and hepatic artery were involved as proximally as possible. The liver injury in all animals was created by the same investigator for consistency. The bleeding livers were treated randomly with pre-weighed Flashclot $(n=6)$, microparticles $(n=6)$, or tranexamic acidloaded microparticles $(n=6)$, and followed by application of direct pressure immediately until the bleeding stopped. The weights of the exscinded liver anterior extremity were recorded. The bleeding time was defined as the time from the start of the application of the hemostatic agent to the bleeding surface to the time bleeding stopped completely. 


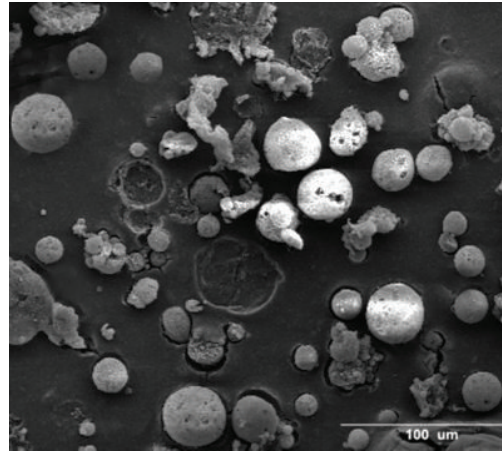

(a)

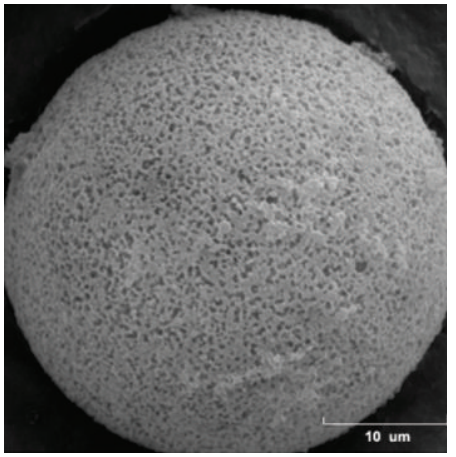

(b)

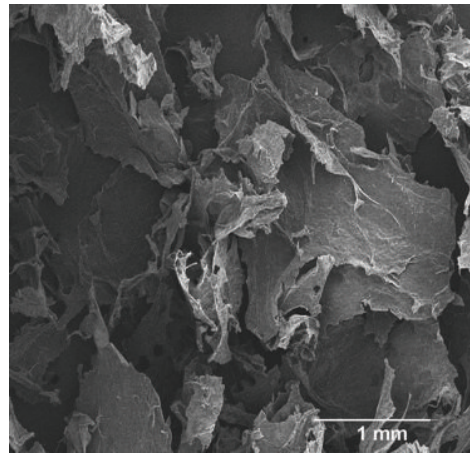

(c)

FIGURE 1: SEM micrograph of microparticles (a) (200x) and (b) (4000x) prepared with emulsification and cross-linking method; (c) prepared by ionic gelation method.

The blood in the abdominal cavity was collected with a preweighed cotton ball. After the bleeding completely stopped, shed blood embedded in the cotton ball and the dressings were collected and weighed to estimate total blood loss. The density of the blood was assumed to be equal to $1.0 \mathrm{~g} / \mathrm{cm}^{3}$. The weight of the blood loss was determined according to the difference between the initial and the final weights of both the cotton ball and the dressings divided by the weight of the animals. Tissue sample was transected from the treated liver edge and preserved in $4 \%$ formalin for histology analysis (paraffin imbedding, HE staining). Surviving rabbits were humanely sacrificed.

2.10. Statistical Analysis. All statistical evaluations were performed using SPSS software. Data were given as mean \pm SD, and differences between groups were compared using a twotailed $t$-test. $P<0.05$ was considered significantly different.

\section{Results}

3.1. Preparation and Characterization of the Microparticles. At first, in order to fabricate the microparticles through a simple procedure, the ionic gelation method was used as previously described in literature [22]. However the microparticles obtained using this method were flattened and leaf-shaped. The surface appeared smooth and slightly porous (Figure 1(c)). On the contrary, through the technology of emulsification and cross-linking, the microparticles obtained possessed a porous surface. Observation under SEM found that the microparticles were spherical, with many micropores distributed over their surfaces (Figures 1(a), 1(b)). Measurement with the powder size analyzer revealed that the microparticles' sizes were $2 \sim 40 \mu \mathrm{m}$ (Figure 2).

The FTIR spectra are shown in Figure 3. Compared to the IR spectra of pure chitosan (Figure 3(a)) and pure alginate (Figure 3(b)), most specific peaks of chitosan and alginate appeared in the spectrum of microparticles (Figure 3(c)) but with some shifts. The characteristic peak of chitosan at $1653 \mathrm{~cm}^{-1}$ corresponded to an amide shift to $1609 \mathrm{~cm}^{-1}$ in spectrum Figure 3(c), but the peak of $-\mathrm{NH}_{2}\left(1595 \mathrm{~cm}^{-1}\right)$

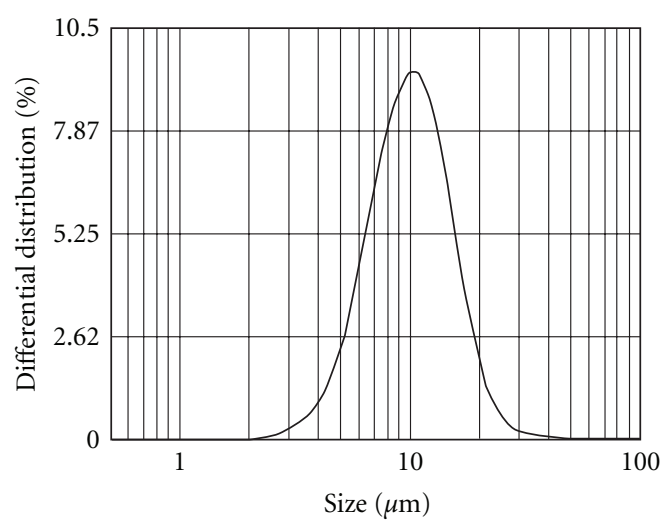

Figure 2: Size distribution of microparticle.

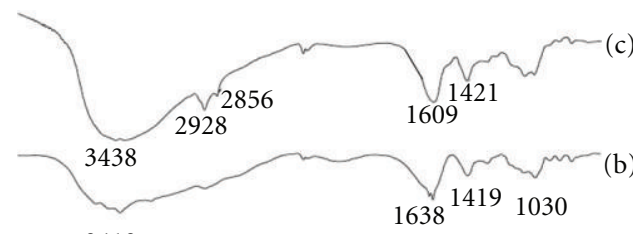

3413

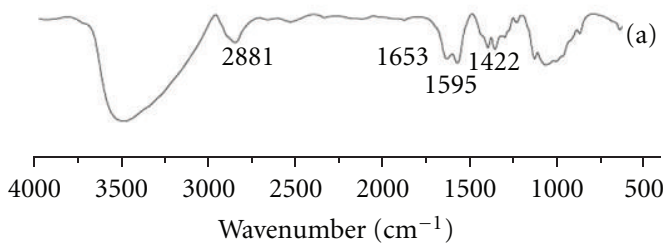

FIGURE 3: IR spectra of (a) pure chitosan, (b) pure sodium alginate, (c) chitosan/alginate composite microparticles.

was absent, which may be the result of the formation of $-\mathrm{NH}_{3}{ }^{+}$. However, the peak at $1421 \mathrm{~cm}^{-1}$ corresponding to the asymmetrical stretching of $-\mathrm{COO}^{-}$was still present. These changes in the IR spectra suggested that there were intermolecular interactions among chitosan and alginate by the electrostatic attraction. 


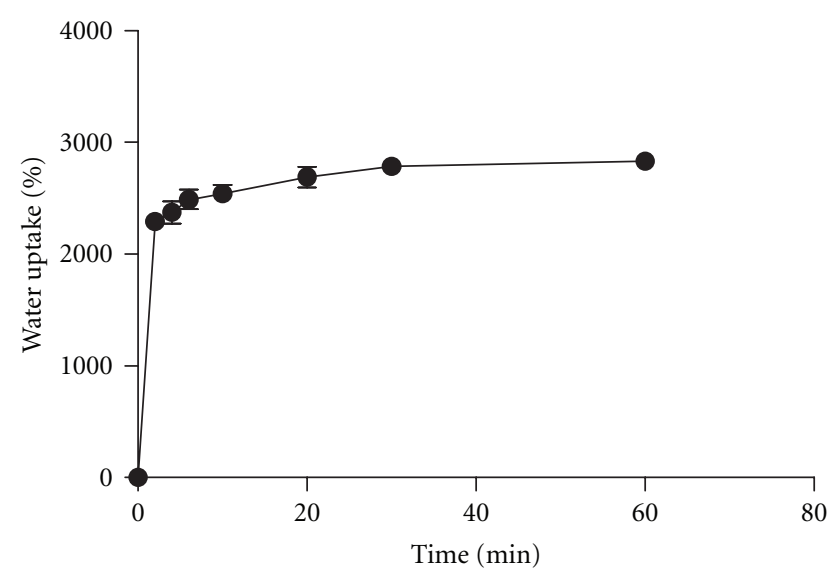

FIGURE 4: Water uptake curve of chitosan/alginate microparticle in SBF. Each data point represents the mean of 3 samples \pm SD.

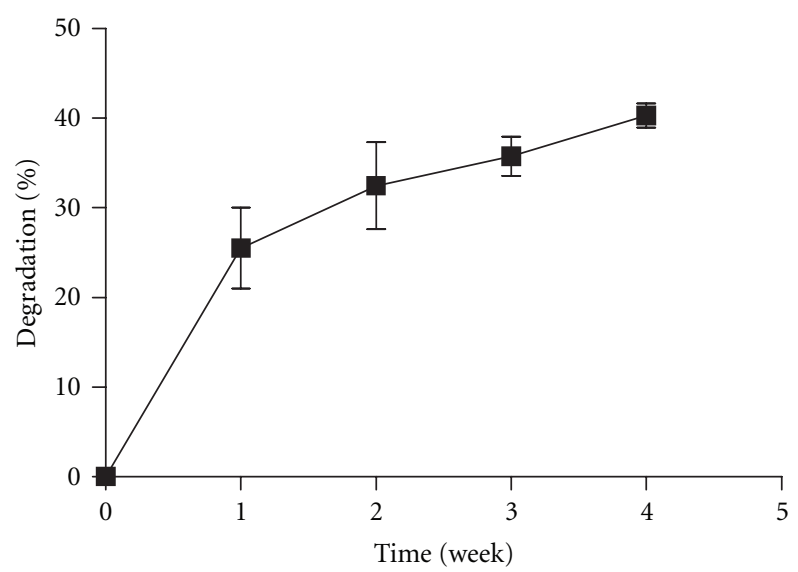

FIGURE 5: In vitro degradation curves of chitosan/alginate microparticle in SBF. Each data point represents the mean of 3 samples \pm SD.

3.2. Water Absorption of the Powder. As shown in Figure 4, the microparticles quickly swelled, and the water absorption ratio rapidly increased within the first $6 \mathrm{~min}$. For example, after soaking for $2 \mathrm{~min}$, the water uptake ratio of the microparticles quickly reached $2293 \%$; then the water uptake ratio gradually increased with time and nearly reached saturation with a water uptake ratio of $2784 \%$ after half an hour. However, the water uptake ratio of the microparticles prepared using the gelation method was lower than 2000\% (data not shown).

3.3. In Vitro Degradation. The percentage of weight loss of the microparticles as a function of degradation time is presented in Figure 5. We observed that the weight loss was faster at the very beginning, and then the degradation ratio gradually increased with incubation time. After 4 weeks, the degradation ratios reached $40 \%$.

3.4. Cytotoxicity. The MTT assay result is shown in Figure 6. Compared with the control group, during the initial period

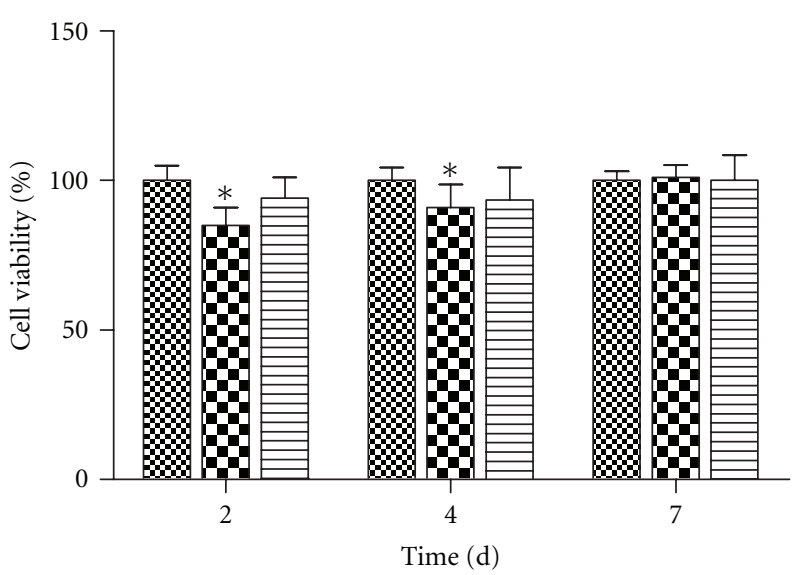

Bedium control
100\% extract
50\% extract

Figure 6: Cytotoxicity of microparticle measured by MTT assay. Each data point represents the mean of 8 samples \pm SD, *: compared with control $P<0.05$.

of cell culture, the $100 \%$ extract group and 50\% extract group displayed a slight inhibition for cell proliferation with $78.1 \%$ and $86.1 \%$ cells viability, respectively. However, the inhibition faded with the extract concentration decrease and culture time extension. On day 7 , the relative proliferation rate of the $100 \%$ extract group reached $100 \%$.

As shown in Figure 7, the number of cells increased with culture time, but no other changes in morphology were observed.

3.5. Drug Release Study. As shown in Figure 8, the release of tranexamic acid from alginate/chitosan microparticles took the form of rapid burst release profiles in both systems. More than $64 \%$ of the initially loaded tranexamic acid was released in $1 \mathrm{~min}$, and the release rate reached $88 \%$ after $3 \mathrm{~min}$. The tranexamic acid was then slowly released during the subsequent time. Comparing curve A with curve B in Figure 8 , the release of tranexamic acid in anticoagulated blood plasma was slightly faster than that in the PBS solution.

3.6. Hemostatic Test. In the hemostatic study, Flashclot was used as the control. All 18 animals completed the study with no exclusions. No significant differences existed among groups for weights, pre-injury MAP, pre-injury blood loss, or the weights of transected livers. During the procedure, no hemodynamic changes were detected that could confound bleeding time (data not shown), and no any rabbit died.

Overall, as shown in Table 1, no obvious diversity in the bleeding time was observed when the wounds were treated with Flashclot or microparticles $(P>0.05)$. However, the bleeding time of the tranexamic acid-loaded microparticle group was significantly shorter than that of the Flashclot group $(P<0.01)$ and the microparticle group $(P<0.05)$. No clear difference in the total blood loss was observed between the three groups. 


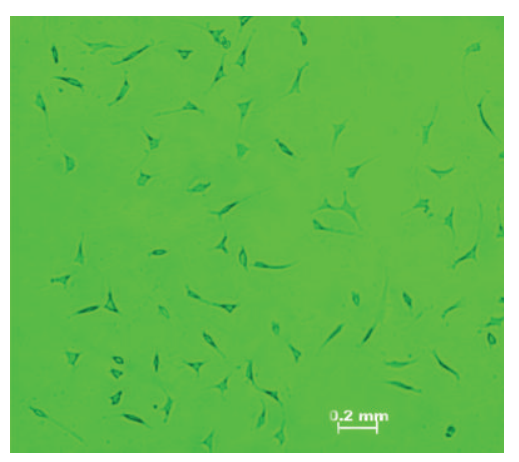

(a)

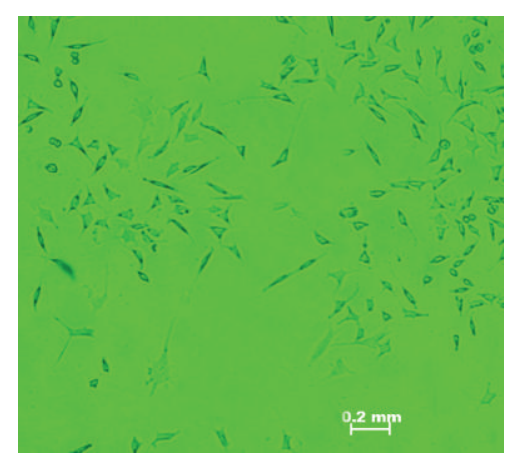

(b)

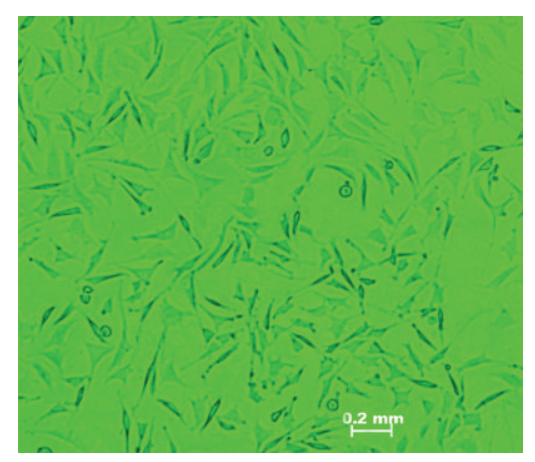

(c)

Figure 7: The morphological observation of the L929 fibroblasts incubated in RPMI-1640 at different culture time (40×): (a) 2 days; (b) 4 days; (c) 7 days.

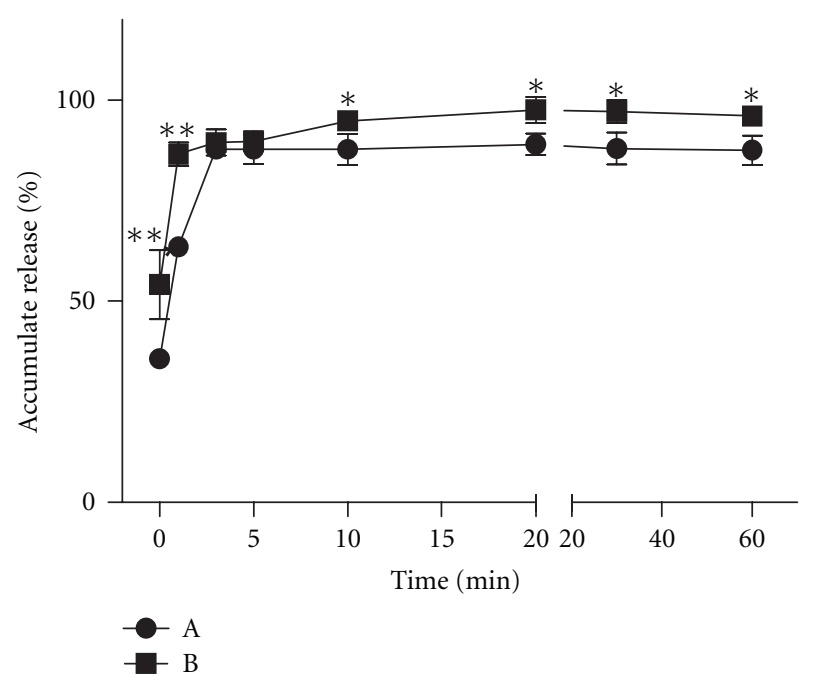

Figure 8: In vitro release profile of tranexamic acid-loaded composite particle A: in PBS (pH 7.4); B: in anticoagulated blood plasma. Each data point represents the mean of 3 samples $\pm S D$; compared with A: ${ }^{*} P<0.05,{ }^{* *} P<0.01$.

During the hemostatic procedure, as we treated the wound with Flashclot and applied a pressure on it, the Flashclot blended with the blood was so hot that we had to cover the Flashclot with several layers of gauzes, but we did not feel the heat when the wound was treated with microparticles. The histology evaluation is shown in Figure 9. In FlashclotTM group, coagulative necrosis in superficial layer contacted with FlashclotTM, remarkable shrinkage and hepatic sinuses dilation in hepatic tissue were observed (Figure 9(b)).

\section{Discussion}

In order to achieve satisfactory hemostatic efficiency, the selection of proper components for preparation of the powdery hemostatic agent is critical. In this study, we chose sodium alginate and chitosan as base materials, not only because they have good properties, such as biocompatibility and biodegradation, but also because they both have certain hemostatic abilities with different hemostatic mechanisms. Moreover, chitosan is a natural cationic polysaccharide polymer; the cationic nature and high charge density of chitosan in solution allows it to form stable ionic complexes with multivalent water-soluble anionic polymers under mild physiological conditions [22, 26]. Alginate is an anionic polysaccharide polymer that can form complexes with polycations such as calcium, chitosan, and polylysine $[27,28]$.

The chitosan-alginate microparticles can be prepared using the gelation method or by emulsification and crosslinking technology. The driving force for the microparticle formation in the gelation method is the decrease in solubility. The mutual neutralization between oppositely charged alginate and chitosan decreases the solubility of the entire system. On the other hand, the guluronic acid moieties of alginate interact with calcium ions to form so-called "egg-box" structures, in which two adjacent macromolecules are linked by means of a physical bond [29]. However, in emulsification and cross-linking, the formation of chitosanalginate microspheres is mainly caused by the differences in their solubility in organic solvents and aqueous solutions.

Due to the porous structure, which can retain a significant amount of water within the pores, the water uptake ability of the microparticles prepared in this study was much better than that of the microparticles prepared by the gelation method.

The cytotoxicity of the microspheres was evaluated in L929 mouse fibroblast cells. As the main cellular components of connective tissues, fibroblast cells are widely used in cytotoxicity studies of biomaterials [30]. In order to avoid confounding factors resulting from the physical trauma of the microspheres, the extract dilution method was chosen over the direct exposure method [31]. We think that the inhibition of the extract on the cell proliferation during the initial period may be due to the leakage of micro-amounts of organic compounds which remained in the microsphere. However, owing to the promotion of cell proliferation by chitosan [32], with the increase of culture time, the influence of micro-amounts organic compounds was counteracted, and the cells recovered gradually. Thus, the relative cell 
TABLE 1: The hemostatic efficiency of several materials for the hepatic hemorrhage model of rabbits.

\begin{tabular}{lccc}
\hline & Weight of resected liver $(\mathrm{g})$ & Total blood loss $(\mathrm{g} / \mathrm{kg})$ & Bleeding time $(\mathrm{min})$ \\
\hline Flashclot & $1.98 \pm 0.22$ & $1.61 \pm 0.83$ & $3.07 \pm 0.84$ \\
Composite particle & $1.93 \pm 0.27$ & $1.27 \pm 0.47$ & $2.48 \pm 0.88$ \\
Tranexamic acid-loaded particle & $1.84 \pm 0.21$ & $1.32 \pm 0.63$ & $1.90 \pm 0.75^{\Delta *}$ \\
\hline
\end{tabular}

${ }^{\Delta} P<0.01$ compared with Flashclot, ${ }^{*} P<0.05$ compared with composite powder.

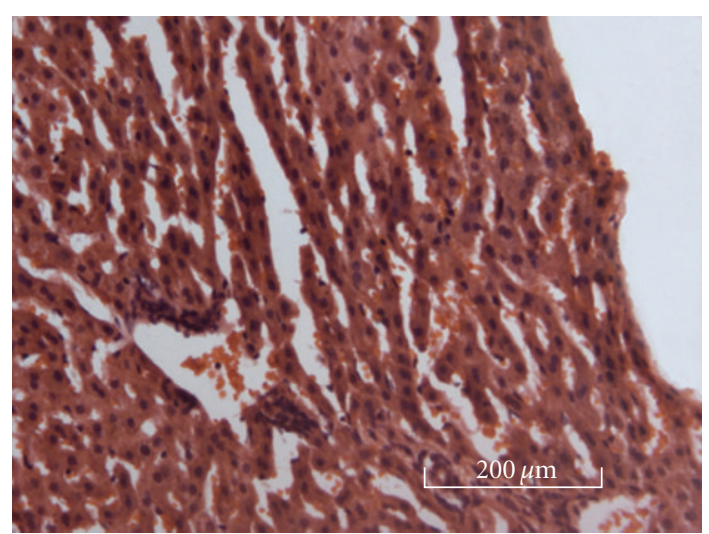

(a)

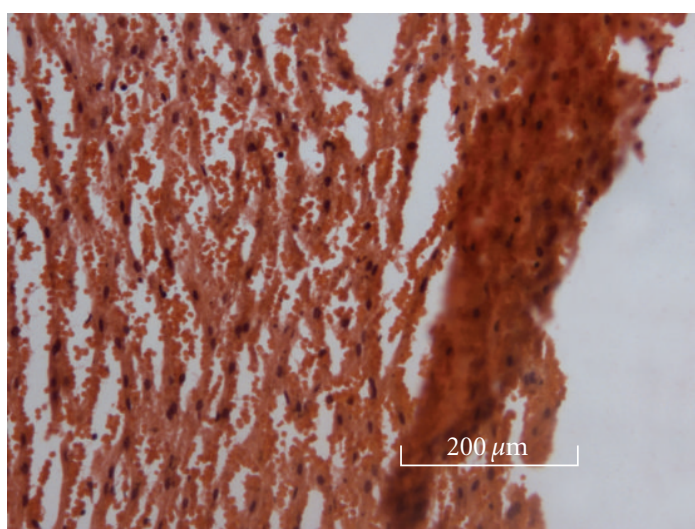

(b)

FIGURE 9: HE- stained histological image $(\times 200)$, (a) microparticles group; (b) Flashclot group.

proliferation rate reached $100 \%$ in later periods of cell culture.

The use of chitosan and sodium alginate carriers as drug delivery systems has gained wide interest [33-35]. Generally, drug release from carrier is affected by many factors, such as the properties of materials used in the carrier, preparation method of the carrier, and $\mathrm{pH}$ values and ionic strength of the release media. First, the drug-loaded particles were prepared by dissolved tranexamic acid in the chitosan/alginate colloidal solution. Then, the mixture was added drop-wise to petroleum, and with the formation of the particles, tranexamic acid was encapsulated in the particles. Interestingly, we found that most of the tranexamic acid was removed during the subsequent purification process. Therefore, we initially prepared porous composite particles with chitosan and sodium alginate, and then using physical absorption, the tranexamic acid was loaded on the composite particle.

Because the particles come in contact mainly with blood when applied to wounds, and the composition of blood can affect the release of tranexamic acid, we carried out the drug release tests in both PBS and anticoagulated blood plasma. The initial burst release clearly revealed that the tranexamic acid was absorbed mainly on the surface of the microspheres, and the porous structure of the particles facilitated the release of tranexamic acid. However, the burst release of tranexamic acid was exactly what we expected, because high doses of tranexamic acid are required in direct hemostatic application. Owing to the fact that the $\mathrm{pH}$ value of the PBS solution used in this study was almost equal to that of anticoagulated blood plasma (7.4), but the ionic strength of both systems was different, we attributed the diversity of tranexamic acid release in both systems to the ionic strength and the influence of proteins in the plasma. However, identifying the detailed mechanism behind this will require further research.

Flashclot is developed by the Honghua Pharmaceutical Co. Ltd and the Fourth Military Medical University. Because its hemostatic performance is similar to Quickclot, Flashclot is also defined as quick-acting styptic powder [36]. Flashclot is made of zeolite, which has a porous structure. In this paper, Flashclot was used as the control to evaluate the hemostatic performance of alginate/chitosan microspheres. As mentioned previously, the alginate/chitosan microparticles also have many small pores on their surfaces. When applied to wounds, the micropores can quickly absorb the water from blood and cause the aggregation of platelets, blood coagulation factors, fibrins, red blood cells, and blood clotting factors to the surface of particles to form a blood clot, and to seal the crevasse of blood vessels. In this study, unlike Flashclot, the alginate/chitosan microspheres did not generate heat when absorbing water from blood and did not result in reinjury of the treated liver.

In addition to the water absorbing ability of the micropores, the positive charge on the surface of chitosan can attract red blood cells and platelets and enhance the adhesion and aggregation of platelets, which results in the formation of a cell embolus or thrombus and promotes blood clotting. In turn, the increase of the local concentration of platelets, blood coagulation factors, and fibrin initiate and enhance the internal clotting mechanism [37, 38].

In addition to the activation of the blood clotting system, it is well known that when the tissues of the endomembrane are exposed after the cutting of blood vessels, the fibrinolytic system is also activated. Unfortunately, the constructed 
thrombus can be dissolved over time. Tranexamic acid is a local hemostatic agent, which can inhibit the degradation of fibrin by inhibiting plasminogen activation which forms fibrinolytic enzymes. Thus, in this study, as the tranexamic acid-loaded composite particles were used on the wound, the porous composite particles enhanced the formation of the thrombus. In addition, the tranexamic acid released from the particles inhibited the degradation of the thrombus. Due to the synergistic effects of several hemostatic mechanisms, the tranexamic acid loaded composite particles exhibited an improved hemostatic efficiency.

\section{Conclusions}

In this study, a new kind of microparticle was prepared based on chitosan and sodium alginate and using emulsification and cross-linking. These spherical microparticles had a porous structure, confined size distribution, satisfactory water absorption, and acceptable biodegradability and safety. Through loading of a local hemostatic agent, tranexamic acid, the hemostatic performance of the microparticles was clearly improved. Importantly, the powdery hemostatic agent not only demonstrated good hemostatic performance and could be used in any wound with irregular depth or geometry, but also should not result in re-injury to the wounded tissues as Flashclot does. Furthermore, due to its biodegradability and safety, this agent could be used to treat intra-abdominal solid organ injuries as well as external wounds. In addition, the powder is ready and easy to use with minimal training, and it is lightweight and durable. Given these characteristics, the microparticles appear simple and effective for use as a hemostatic agent. However, further studies will be needed to understand the hemostatic efficiency of the microparticles in aortic bleeding and in coagulopathic subjects compared with many other currently available dreesings.

\section{Conflict of Interests}

The authors declare that they have no conflict of interests.

\section{Acknowledgments}

The authors wish to thank the State Key Laboratory of Trauma, Burns, and Combined Injury for their funding support. Contract Gant sponsor: State Key Laboratory of Trauma, Burns and Combined Injury, contract grant number: SKLZZ200812.

\section{References}

[1] H. R. Champion, R. F. Bellamy, C. P. Roberts, and A. Leppaniemi, "A profile of combat injury," Journal of Trauma, vol. 54, no. 5, pp. S13-S19, 2003.

[2] A. Sauaia, F. A. Moore, E. E. Moore et al., "Epidemiology of trauma deaths: a reassessment," Journal of Trauma, vol. 38, no. 2, pp. 185-193, 1995.

[3] A. E. Pusateri, J. B. Holcomb, B. S. Kheirabadi, H. B. Alam, C. E. Wade, and K. L. Ryan, "Making sense of the preclinical literature on advanced hemostatic products," The Journal of Trauma, vol. 60, no. 3, pp. 674-682, 2006.

[4] H. B. Alam, D. Burris, J. A. Dacorta, and P. Rhee, "Hemorrhage control in the battlefield: role of new hemostatic agents," Military Medicine, vol. 170, no. 1, pp. 63-69, 2005.

[5] J. N. Vournakis, M. Demcheva, A. B. Whitson, S. Finkielsztein, and R. J. Connolly, "The RDH Bandage: hemostasis and survival in a lethal aortotomy hemorrhage model," Journal of Surgical Research, vol. 113, no. 1, pp. 1-5, 2003.

[6] B. S. Kheirabadi, E. M. Acheson, R. Deguzman et al., "The potential utility of fibrin sealant dressing in repair of vascular injury in swine," Journal of Trauma, vol. 62, no. 1, pp. 94-103, 2007.

[7] S. H. Baik, J. H. Kim, H. H. Cho, S. N. Park, Y. S. Kim, and H. Suh, "Development and analysis of a collagen-based hemostatic adhesive," Journal of Surgical Research, vol. 164, no. 2, pp. e221-e228, 2010.

[8] A. E. Pusateri, J. B. Holcomb, B. S. Kheirabadi, H. B. Alam, C. E. Wade, and K. L. Ryan, "Making sense of the preclinical literature on advanced hemostatic products," Journal of Trauma, vol. 60, no. 3, pp. 674-682, 2006.

[9] A. E. Pusateri, A. V. Delgado, E. J. Dick, R. S. Martinez, J. B. Holcomb, and K. L. Ryan, "Application of a granular mineralbased hemostatic agent (QuikClot) to reduce blood loss after grade V liver injury in swine," Journal of Trauma, vol. 57, no. 3, pp. 555-562, 2004.

[10] H. B. Alam, Z. Chen, A. Jaskille et al., "Application of a zeolite hemostatic agent achieves 100\% survival in a lethal model of complex groin injury in swine," Journal of Trauma, vol. 56, no. 5, pp. 974-983, 2004.

[11] J. K. Wright, J. Kalns, E. A. Wolf et al., "Thermal injury resulting from application of a granular mineral hemostatic agent," Journal of Trauma, vol. 57, no. 2, pp. 224-230, 2004.

[12] T. R. Hurtado and T. Wisenbaugh, "Images in emergency medicine: superficial partial-thickness (second-degree) burn from zeolite mineral hemostatic agent (QuickClot [Z-Medica, Newington, CT])," Annals of Emergency Medicine, vol. 46, no. 3, pp. 297-303, 2005.

[13] T. Dai, G. P. Tegos, M. Burkatovskaya, A. P. Castano, and M. R. Hamblin, "Chitosan acetate bandage as a topical antimicrobial dressing for infected burns," Antimicrobial Agents and Chemotherapy, vol. 53, no. 2, pp. 393-400, 2009.

[14] D. Raafat, K. Von Bargen, A. Haas, and H. G. Sahl, "Insights into the mode of action of chitosan as an antibacterial compound," Applied and Environmental Microbiology, vol. 74, no. 12, pp. 3764-3773, 2008.

[15] M. Burkatovskaya, G. P. Tegos, E. Swietlik, T. N. Demidova, A. P Castano, and M. R. Hamblin, "Use of chitosan bandage to prevent fatal infections developing from highly contaminated wounds in mice," Biomaterials, vol. 27, no. 22, pp. 4157-4164, 2006.

[16] A. K. Azad, N. Sermsintham, S. Chandrkrachang, and W. F. Stevens, "Chitosan membrane as a wound-healing dressing: characterization and clinical application," Journal of Biomedical Materials Research B, vol. 69, no. 2, pp. 216-222, 2004.

[17] K. Kojima, Y. Okamoto, K. Kojima et al., "Effects of chitin and chitosan on collagen synthesis in wound healing," Journal of Veterinary Medical Science, vol. 66, no. 12, pp. 1595-1598, 2004.

[18] A. E. Pusateri, S. J. McCarthy, K. W. Gregory et al., "Effect of a chitosan-based hemostatic dressing on blood loss and survival in a model of severe venous hemorrhage and hepatic injury in swine," Journal of Trauma, vol. 54, no. 1, pp. 177-182, 2003. 
[19] V. Y. Sohn, M. J. Eckert, M. J. Martin et al., "Efficacy of three topical hemostatic agents applied by medics in a lethal groin injury model," Journal of Surgical Research, vol. 154, no. 2, pp. 258-261, 2009.

[20] S. Y. Ong, J. Wu, S. M. Moochhala, M. H. Tan, and J. $\mathrm{Lu}$, "Development of a chitosan-based wound dressing with improved hemostatic and antimicrobial properties," Biomaterials, vol. 29, no. 32, pp. 4323-4332, 2008.

[21] R. L. Gardner, "Application of alginate gels to the study of Mammalian development," Methods in Molecular Biology, vol. 254, pp. 383-392, 2004.

[22] M. Lee, W. Li, R. K. Siu et al., "Biomimetic apatite-coated alginate/chitosan microparticles as osteogenic protein carriers," Biomaterials, vol. 30, no. 30, pp. 6094-6101, 2009.

[23] T. Kokubo and H. Takadama, "How useful is SBF in predicting in vivo bone bioactivity?" Biomaterials, vol. 27, no. 15, pp. 2907-2915, 2006.

[24] Y. Liu, C. Hou, and Q. Gu, "Preparation and evaluation of chitosan/alginate microsphere as a novel biodegradable haemostatic powder," Zhongguo Xiu Fu Chong Jian Wai Ke Za Zhi, vol. 21, no. 8, pp. 829-832, 2007.

[25] China Standards, "Biological evaluation of medical devicespart 5: test for in vitro cytotoxicity," GB/T, 16886. 5-2003.

[26] X. Z. Shu and K. J. Zhu, "A novel approach to prepare tripolyphosphate/chitosan complex beads for controlled release drug delivery," International Journal of Pharmaceutics, vol. 201, no. 1, pp. 51-58, 2000.

[27] M. L. González-Rodríguez, M. A. Holgado, C. SánchezLafuente, A. M. Rabasco, and A. Fini, "Alginate/chitosan particulate systems for sodium diclofenac release," International Journal of Pharmaceutics, vol. 232, no. 1-2, pp. 225-234, 2002.

[28] T. A. Becker, D. R. Kipke, and T. Brandon, "Calcium alginate gel: a biocompatible and mechanically stable polymer for endovascular embolization," Journal of Biomedical Materials Research A, vol. 54, pp. 76-86, 2001.

[29] L. Oddo, G. Masci, C. Di Meo et al., "Novel thermosensitive calcium alginate microspheres: physico-chemical characterization and delivery properties," Acta Biomaterialia, vol. 6, no. 9, pp. 3657-3664, 2010.

[30] S. K. Bhatia and A. B. Yetter, "Correlation of visual in vitro cytotoxicity ratings of biomaterials with quantitative in vitro cell viability measurements," Cell Biology and Toxicology, vol. 24, no. 4, pp. 315-319, 2008.

[31] R. De Souza, P. Zahedi, C. J. Allen, and M. PiquetteMiller, "Biocompatibility of injectable chitosan-phospholipid implant systems," Biomaterials, vol. 30, no. 23-24, pp. 38183824, 2009.

[32] G. I. Howling, P. W. Dettmar, P. A. Goddard, F. C. Hampson, M. Dornish, and E. J. Wood, "The effect of chitin and chitosan on the proliferation of human skin fibroblasts and keratinocytes in vitro," Biomaterials, vol. 22, no. 22, pp. 2959 2966, 2001.

[33] S. F. Nie, X. M. Wu, H. F. Liu, H. W. Jiang, and W. S. Pan, "Studies on the influencing factors on the drug release from sodium alginate matrices," Yaохue Xuebao, vol. 39, no. 7, pp. 561-565, 2004.

[34] P. Matricardi, C. Di Meo, T. Coviello, and F. Alhaique, "Recent advances and perspectives on coated alginate microspheres for modified drug delivery," Expert Opinion on Drug Delivery, vol. 5, no. 4, pp. 417-425, 2008.

[35] J. H. Hamman, "Chitosan based polyelectrolyte complexes as potential carrier materials in drug delivery systems," Marine Drugs, vol. 8, no. 4, pp. 1305-1322, 2010.
[36] R. Zhou, Z. Huang, X. Cheng et al., "Clinical research on the treatment of acute trauma hemorrhage with quick-acting styptic powder," China Journal Critical Care Medical, vol. 27, no. 6, pp. 521-524, 2007.

[37] Y. Okamoto, R. Yano, K. Miyatake, I. Tomohiro, Y. Shigemasa, and S. Minami, "Effects of chitin and chitosan on blood coagulation," Carbohydrate Polymers, vol. 53, no. 3, pp. 337$342,2003$.

[38] J. Zhang, W. Xia, P. Liu et al., "Chitosan modification and pharmaceutical/biomedical applications," Marine Drugs, vol. 8, no. 7, pp. 1962-1987, 2010. 

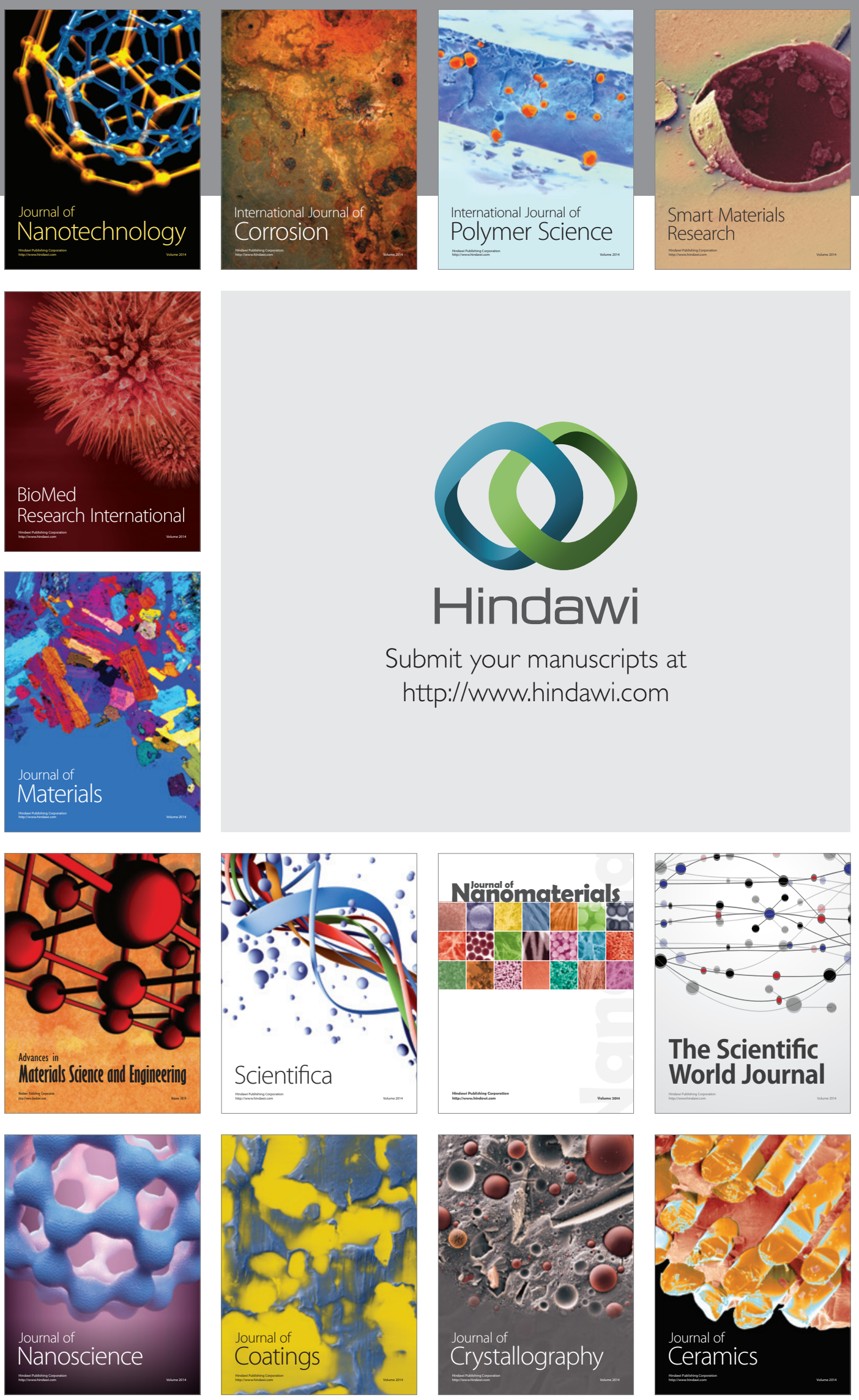

The Scientific World Journal

Submit your manuscripts at

http://www.hindawi.com

\section{World Journal}

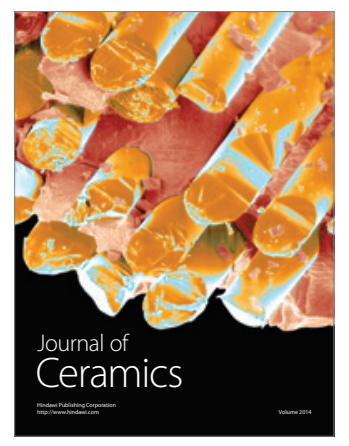

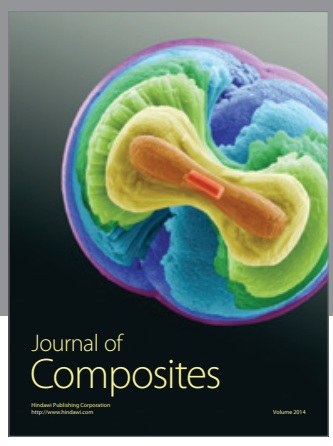
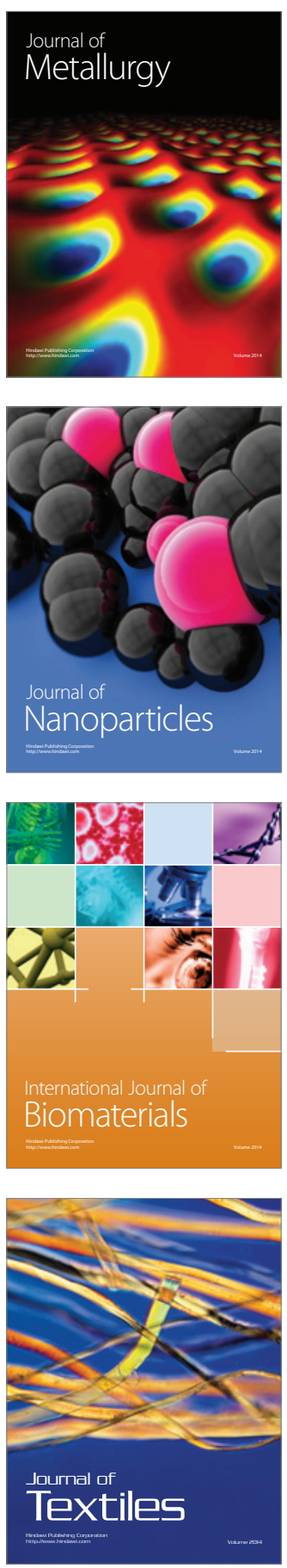